\title{
A simple and efficient electrochemical reductive method for graphene oxide
}

\author{
YANYUN LIU, DONG ZHANG*, YU SHANG and CHAO GUO \\ School of Material Science and Engineering, Tongji University, Caoan Road, Shanghai 200092, \\ People's of Republic of China
}

MS received 29 July 2013; revised 1 October 2013

\begin{abstract}
The electrochemical reduction of graphene oxide typically involves complicated procedures, such as modification of electrodes and preparation of electrolytes, which is often needed in previous reports. In this paper, a simple and efficient electrochemical process is described for the synthesis of high-quality reduced graphene oxide. The main procedures involve the electrophoretic deposition of graphene oxide onto positive electrode and the subsequent in situ electrochemical negative reduction when the electrode changes from positive to negative. This approach opens up a new, practical and green reducing method to prepare largescale graphene.
\end{abstract}

Keywords. Graphene oxide; electrophoretic deposition; negative reduction.

\section{Introduction}

The synthesis of graphene has been one of the hottest topics in scientific community in recent years because of its remarkable physical and chemical properties (Novoselov et al 2004; Lee et al 2008; Geim 2009) and its great promising application in devices or composites (Qu et al 2010; Wu et al 2010; Zhu et al 2010). During the past years, diverse procedures have been established for producing graphene, such as mechanical exfoliation (Novoselov et al 2004), epitaxial growth (Emtsev et al 2009; Kim et al 2009) and chemical or thermal reduction of graphene oxide (GO) (Stankovich et al 2007; Fan et al 2008). The synthesis route from graphite oxide is considered to be the most economical way for mass production of graphene (Geim et al 2009). However, it often employs hazardous chemicals (e.g. hydrazine) as reductants or rapid heat treatment at high temperature. The 'green synthesis' of graphene under mild conditions is preferred (Fan et al 2008). Electrochemical methods are a promising green strategy for graphene synthesis, and several research works have been reported (Ramesha and Sampath 2009; Zhou et al 2009). For example, An et al (2010) prepare graphene thin films by electrophoretic deposition of GO and simultaneous positive reduction. Zhou et al (2009) synthesized graphene films by coupling negative reduction and spray coating in various electrolytes. However, these methods would suffer from the limits of tedious procedures or want of special equipment. It is not suitable for large-scale production.

\footnotetext{
*Author for correspondence (zhangdng@tongji.edu.cn)
}

Moreover, the obtained products existed in the form of films absorbed on the electrode, which made the output very less efficient. Therefore, it is imperative to explore a simpler and more efficient electrochemical method to synthesize graphene.

In this paper, a simple and an efficient electrochemical process is described for the synthesis of graphene. The main procedures involve the electrophoretic deposition of GO on to positive electrode and the subsequent in situ electrochemical negative reduction when the electrode changes from positive to negative. Compared with the reported electrochemical reduction methods, our method has the following significant advantages: (1) It is simple to operate. In this method, several complicated procedures such as modification of electrodes and preparation of electrolytes are not needed. (2) It is highly efficient. The products can be collected not just from electrode, but also from the solution. It provides a promising method for large-scale fabrication of graphene.

\section{Experimental}

The GO used in this study was synthesized by the modified Hummers and Offeman (1958) method. It was dispersed in water $(1 \mathrm{mg} / \mathrm{mL})$ and sonicated for $2 \mathrm{~h}$ at room temperature. As much as $50 \mathrm{~mL}$ GO aqueous dispersion $\left(1 \mathrm{mg} \mathrm{mL}^{-1}\right)$ was loaded in a $75 \mathrm{~mL}$ glass container. Copper electrodes were used in the experiment. The electrode separation was $5 \mathrm{~mm}$. Figure 1 shows the electrochemical reaction process. The GO platelets migrated towards the positive electrode when a direct current (d.c.) voltage was applied. The adsorptive voltage and absorptive time were $30 \mathrm{~V}$ 


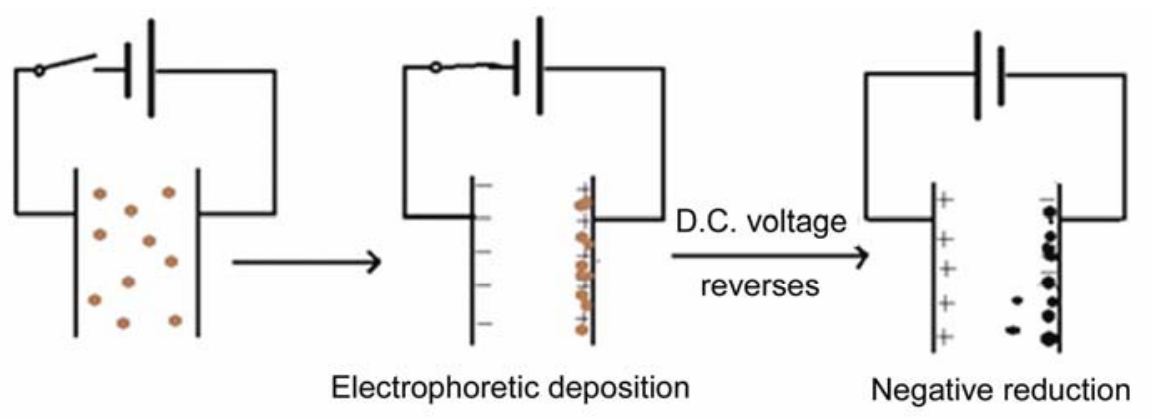

Figure 1. Schematic illustration of electrochemical reaction.

Table 1. Samples prepared at different reductive voltages and time.

\begin{tabular}{lcc}
\hline Sample & Reductive voltage (V) & Reductive time (min) \\
\hline a-1 & 60 & 60 \\
a-2 & 60 & 120 \\
a-3 & 60 & 180 \\
a-4 & 60 & 240 \\
b-4 & 45 & 240 \\
c-4 & 30 & 240 \\
\hline
\end{tabular}

and $30 \mathrm{~min}$, respectively. Then, adsorptive GO sheets were in situ reduced at certain reductive voltage and time, when the electrodes changed from positive to negative. The obtained samples were taken out from the solution and the electrode and thoroughly washed with deionized water and dried at $50{ }^{\circ} \mathrm{C}$. We marked the samples as a-1, $\mathrm{a}-2, \ldots, \mathrm{c}-4$ according to different reductive voltage and time as in table 1.

The UV-Visible (UV-Vis) absorption spectra of the GO and graphene suspension were recorded with a Hitachi U-3310 spectrophotometer. The X-ray diffraction (XRD) images of the samples were measured using an X-ray diffractometer (D/max2550VB3+/PC, Rigaku, Japan) with reference target: $\mathrm{CuK} \alpha$ radiation. Fourier transform infrared (FT-IR) spectra were obtained through a Bruker Equinoxss spectrometer use the attenuated total reflectance. X-ray photoelectron spectroscopy (XPS) studies were conducted on an RBD-upgraded PHI-5000C ESCA system (Perkin Elmer) with MgK $\alpha$ radiation ( $h v=$ $1253.6 \mathrm{eV}$ ). Raman scattering (Raman) was performed on an INVIA Raman spectrometer using a $488 \mathrm{~nm}$ laser source.

\section{Results and discussion}

The UV-Vis absorption peak of GO dispersion and the obtained graphene at different times are shown in figure 2(A). The peak of GO around $230 \mathrm{~nm}$ could be ascribed to the K-band absorption of aromatic compounds. It redshifted towards $260 \mathrm{~nm}$ after electrochemical reduction for $60 \mathrm{~min}$ and gradually redshifted to
$270 \mathrm{~nm}$ after $240 \mathrm{~min}$. This suggests that the electronic conjugation within the graphene nanosheets had been mainly restored after 240 min of reduction.

FT-IR spectra of GO dispersion and the obtained graphene at different times are shown in figure 2(B). The most characteristic features of GO are at $1060 \mathrm{~cm}^{-1}$ (C-O stretching vibrations), $1706 \mathrm{~cm}^{-1}$ ( $\mathrm{C}=\mathrm{O}$ stretching vibrations from carbonyl and carboxylic groups), $1650 \mathrm{~cm}^{-1}$ $(\mathrm{C}=\mathrm{C}$ stretching vibrations from unoxidized graphitic domains), $1340 \mathrm{~cm}^{-1}$ (C-H bending vibration) and $3390 \mathrm{~cm}^{-1}$ (O-H stretching vibrations). After $60 \mathrm{~min}$ of reduction, the strongest vibrational absorption bands of $\mathrm{O}-\mathrm{H}$ became weak (figure $2 \mathrm{~B}(\mathrm{a}-1)$. The intensities of the peaks corresponding to the oxygen functionalities all decreased dramatically or even disappeared, when the reductive time reached $240 \mathrm{~min}$ (Figure 2B(a-4). These data reveal that the GO have been successfully reduced with the time increased.

X-ray diffraction (XRD) of GO dispersion and the obtained graphene at different times are shown in figure 2(C). GO exhibited a very sharp diffraction peak at $2 \theta=11 \cdot 4^{\circ}$. After $60 \mathrm{~min}$ of reduction, the intensities of peak became weak at $2 \theta=11 \cdot 4^{\circ}$. Meanwhile, a new peak of graphene at $2 \theta=23.9^{\circ}$, weak and broad, was observed. The regular stacks of GO were destroyed and their diffraction peaks at $2 \theta=11.4^{\circ}$ became weaker or even disappeared, when the reductive time reached $240 \mathrm{~min}$, indicates that the oxygen-containing group of GO had been efficiently removed. Meanwhile, interlayer $d$-spacing became small.

Figure 2(D) shows Raman spectra of GO dispersion and the obtained graphene at different reductive times. In carbonaceous material, the D and G bands correspond to $s p^{2}$ and $s p^{3}$ carbon-stretching modes and their intensity ratio $\left(I_{\mathrm{D}} / I_{\mathrm{G}}\right)$ is a measure of the amount of disorder present within the materials. The bands of GO associated at $\sim 1350$ and $\sim 1610 \mathrm{~cm}^{-1}$ correspond to $\mathrm{D}$ and $\mathrm{G}$ modes, respectively (Kudin et al 2008). The G band starts shifting towards lower wavenumbers, indicating the beginning of the reduction process when the reductive time is changed from 60 to $180 \mathrm{~min}$. It is observed around $\sim 1590 \mathrm{~cm}^{-1}$ after $240 \mathrm{~min}$. The D band also shows a similar variation in its position, from 1350 to $1340 \mathrm{~cm}^{-1}$ 

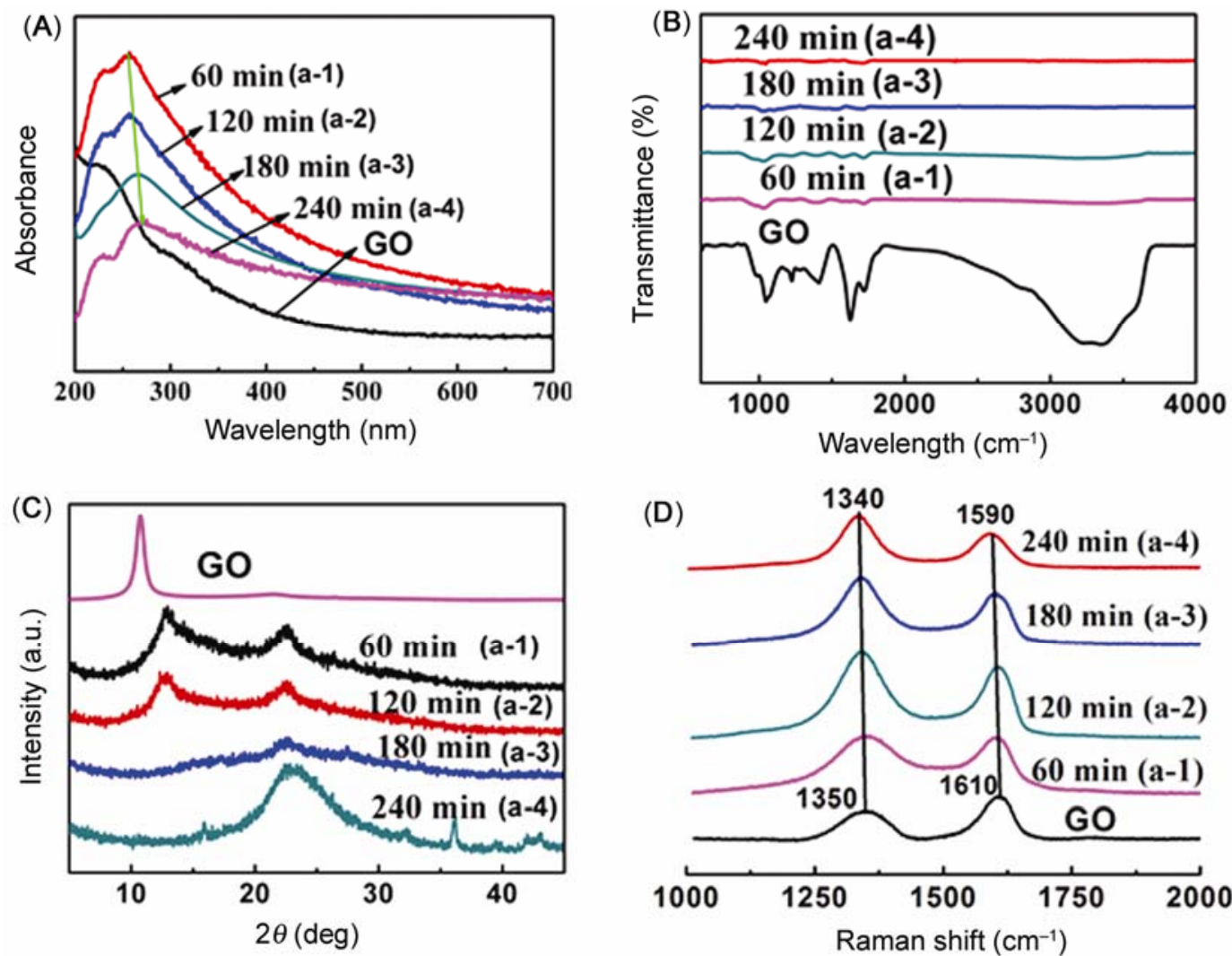

Figure 2. (A) UV-Vis absorption spectra, (B) FT-IR spectra, (C) X-ray diffraction and (D) Raman spectra of GO dispersion and the obtained graphene at $60 \mathrm{~V}$ reductive voltage and different reductive times (a-1: 60 min, a-2: $120 \mathrm{~min}, \mathrm{a}-3: 180 \mathrm{~min}$ and a-4: $240 \mathrm{~min}$ ).

in the same potential range. This is similar to the data reported by Stankovich et al (2007) for the chemical reduction of GO to RGO, The Raman spectra showed an increase in the $D / G$ ratio with the reductive time increased, indicating the increased defects or edge areas from GO to graphene. The defects might be due to the smaller size of graphene sheets (Stankovich et al 2007).

The UV-Vis absorption peak of the obtained graphene at different reductive voltages are shown in figure 3(A). The absorption peak around $267 \mathrm{~nm}$ was found when the reductive voltage was $30 \mathrm{~V}$. Then it gradually redshifted to $270 \mathrm{~nm}$ with the reductive voltage increased to $60 \mathrm{~V}$, indicating that the electronic conjugation is restored. FT-IR spectra of obtained graphene at different reductive voltages are shown in figure 3(B), the intensities of the peaks corresponding to the oxygen functionalities, all decreased dramatically or even disappeared with the reductive voltage increased to $60 \mathrm{~V}$. These data reveal that the GO has been successfully reduced with increase in reductive voltage. XRD images of obtained graphene at different reductive voltages are shown in figure $3(\mathrm{C})$. A very sharp diffraction peak at $2 \theta=11 \cdot 4^{\circ}$ and a weak peak at $23.9^{\circ}$ were found when the reductive voltage was $30 \mathrm{~V}$. The intensities of peak became weak at $2 \theta=11 \cdot 4^{\circ}$, with the reductive voltage increased to $60 \mathrm{~V}$. Meanwhile, a broad peak of graphene at $2 \theta=23.9^{\circ}$ was observed. These give evidence that the graphite oxide was reduced to graphene and as such restored the ordered crystal structure. Figure 3(D) shows the Raman spectra of the obtained graphene at different reductive voltages. The D and $G$ bands are shifted to lower wavenumbers with the reductive voltage increased. For example, the $G$ band is at $1605 \mathrm{~cm}^{-1}$ for $\mathrm{c}-4(30 \mathrm{~V})$, but is at $1590 \mathrm{~cm}^{-1}$ for a-4 $(60 \mathrm{~V})$.

We can infer that sample a- 4 has a better reductive effect compared with other samples from this research. It means that the optimal reductive time and reductive voltage were $240 \mathrm{~min}$ and $60 \mathrm{~V}$, respectively, in our experiment. We can obtain high-quality graphene in that condition. Figure 4(A and B) shows the C 1s X-ray photoelectron spectroscopy (XPS) spectra of GO and sample a-4. Five different peaks centered at $284.7 \quad(\mathrm{C}=\mathrm{C})$, $285.5(\mathrm{C}-\mathrm{C}), 286.8(\mathrm{C}-\mathrm{O}), 287.8(\mathrm{C}=\mathrm{O})$ and $288.9 \mathrm{eV}$ $(\mathrm{O}=\mathrm{C}-\mathrm{OH})$ were detected in the $\mathrm{GO}$ sample. After electrochemical reduction for $240 \mathrm{~min}$, the intensities of all C $1 s$ peaks of the carbons binding to oxygen decreased obviously, revealing that the most oxygen-containing functional groups were removed. The result was consistent with the research above. 

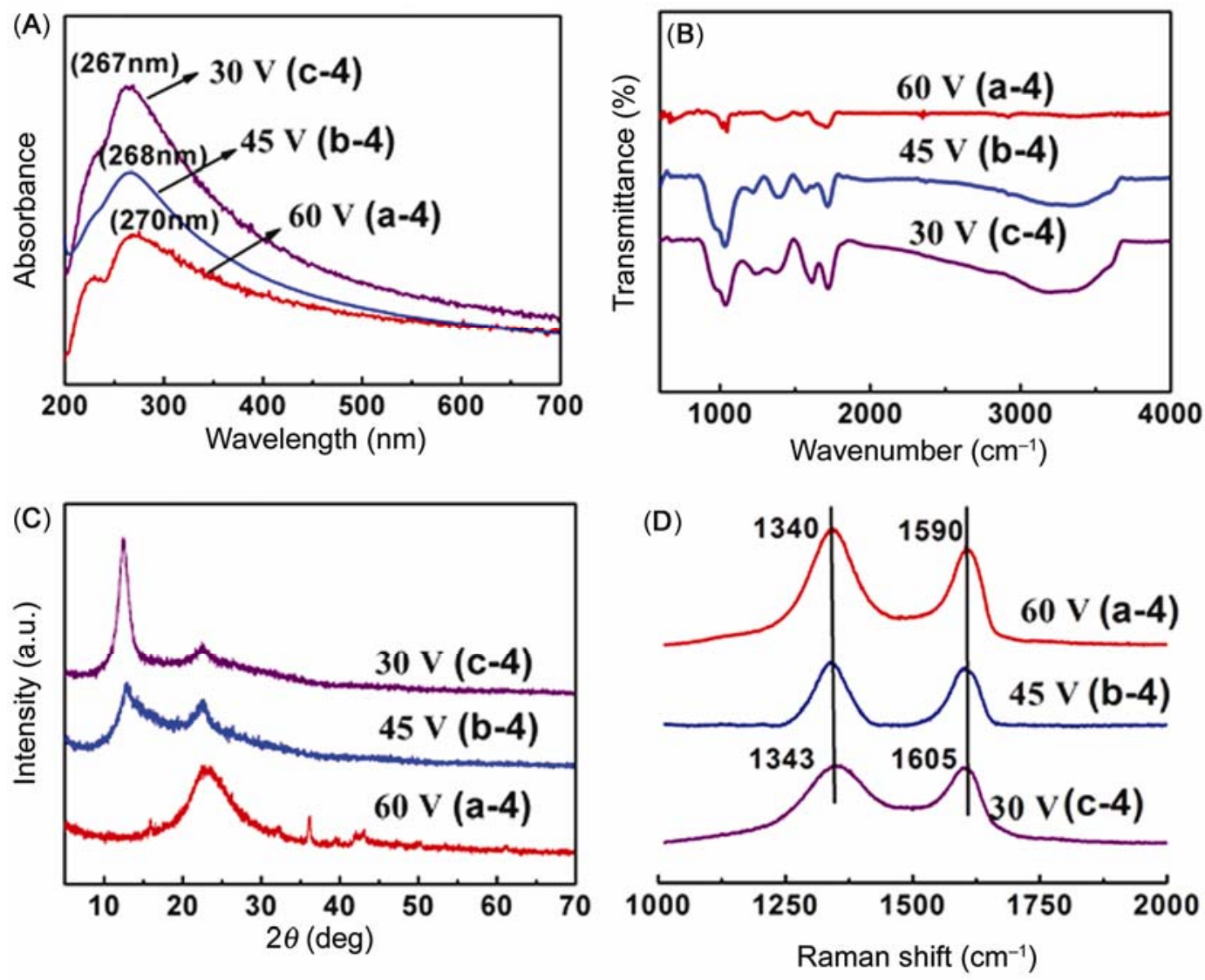

Figure 3. (A) UV-Vis absorption spectra, (B) FT-IR spectra (C) X-ray diffraction and (D) Raman spectra of the obtained graphene at different reductive voltages (a-4: $60 \mathrm{~V}, \mathrm{~b}-4: 45 \mathrm{~V}$ and c-4: $30 \mathrm{~V}$ ) for $240 \mathrm{~min}$.
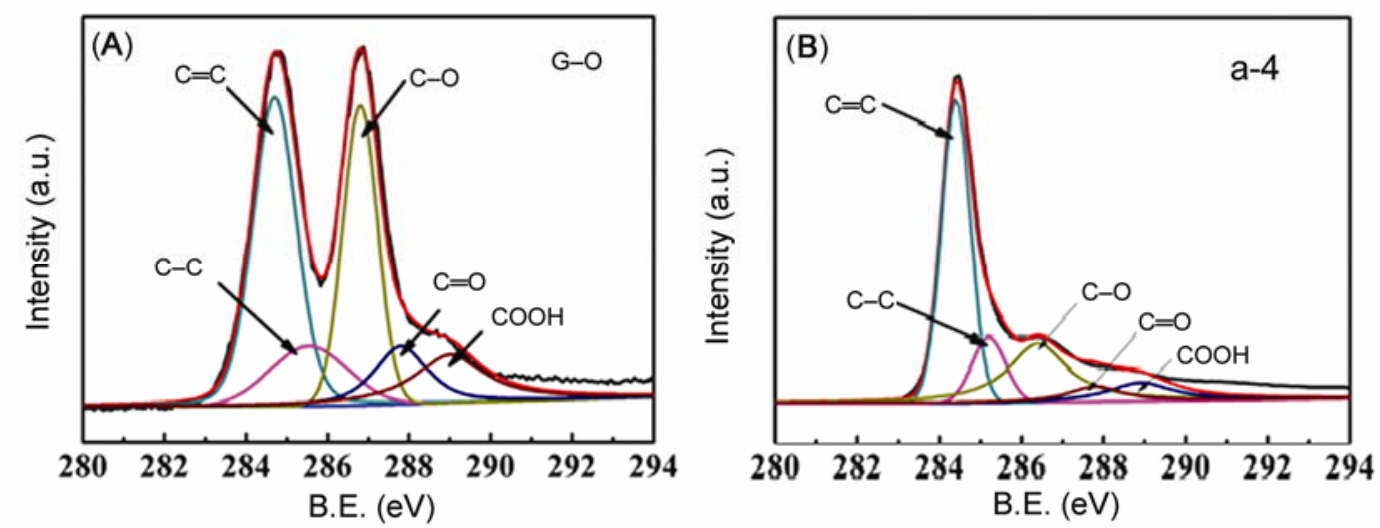

Figure 4. XPS spectra of $\mathrm{C} 1 \mathrm{~s}$ of $\mathrm{GO}$ and sample a-4 (reductive time and reductive voltage were $240 \mathrm{~min}$ and $60 \mathrm{~V}$, respectively).

\section{Conclusions}

In conclusion, this paper provides a simple and an efficient electrochemical method to obtain high-quality graphene. The main procedures involve the electrophoretic deposition of graphene oxide on to positive electrode and the subsequent in situ electrochemical negative reduction, when the electrode changes from positive to negative. The reducing degree of $\mathrm{GO}$ was determined with UVVis absorption spectroscopy, X-ray diffraction, Fourier 
transform infrared spectroscopy and Raman spectra. This approach opens up a new, practical and green reducing method to prepare graphene on a large-scale.

\section{Acknowledgements}

The authors thank the National High Technology Research and Development Program of China (No. 2012AA030303) and Basic Research Key Program of Shanghai (No. 12JC1408600).

\section{References}

An S J, Zhu Y W, Lee S H, Stoller M D, Emilsson T, Park S, Velamakanni A, An J and Ruoff R S 2010 J. Phys. Chem. Lett. 11259

Emtsev K V et al 2009 Nat. Mater. 8203

Fan X B, Peng W C, Li Y, Li X Y, Wang S L, Zhang G L and Zhang F B 2008 Adv. Mater. 204490

Geim A K 2009 Science 3241530
Hong W J, Xu Y X, Lu G W, Li C and Shi G Q 2008 Electrochem. Commun. 101555

Hummers W S and Offeman R E 1958 J. Am. Chem. Soc. 80 1339

Kim K S et al 2009 Nature 457706

Kudin K N, Ozbas B, Schniepp H C, Prud'homme R K, Aksay I A and Car R 2008 Nano Lett. 836

Lee C, Wei X, Kysar J W and Hone J 2008 Science 321385

Novoselov K S, Geim A K, Morozov S V, Jiang D, Zhang Y, Dubonos S V, Crigorieva I V and Firsov A A 2004 Science 306666

Qu L T, Liu Y, Baek J B and Dai L M 2010 ACS Nano 41321

Ramesha G K and Sampath S 2009 J. Phys. Chem. C 1137985

Stankovich S et al 2007 Carbon 451558

Stankovich S, Dikin D A, Piner R D, Kohlhaas K A, Kleinhammes A, Jia Y, Wu Y, Nguyen S T and Ruoff R S 2007 Carbon 451558

Wu Q, Xu Y X, Yao Z Y, Liu A R and Shi G Q 2010 ACS Nano 41963

Zhou M, Wang Y, Zhai Y, Zhai J, Ren W, Wang F and Dong S 2009 Chem. Eur. J. 156116

Zhu Y W, Stoller M D, Cai W W, Velamakanni A, Piner R D, Chen D and Ruoff R S 2010 ACS Nano 41227 